\title{
Uranium and Lead Uptake and Transfer in Plant-Fungi Symbioses
}

\author{
J. DinSLEY ${ }^{1 *}$, C. H. ROBINSON ${ }^{1}$, S. SHAW ${ }^{1}$, L. FIELD ${ }^{2}$ \\ AND J. K. PITTMAN ${ }^{1}$ \\ ${ }^{1}$ Department of Earth and Environmental Sciences, The \\ University of Manchester, Manchester, M13 9PL, UK. \\ ${ }^{2}$ British Geological Survey, Keyworth, Nottingham, NG12 \\ $5 \mathrm{GG}$. \\ (*Corresponding author: james.dinsley@manchester.ac.uk)
}

Land and water pollution from the mining of heavy metals and naturally-occurring radioactive materials (NORMs) poses an expensive and technically difficult challenge to overcome worldwide, resulting in biodiversity loss and the degradation of potential agricultural, commercial or residential land. A more detailed understanding of plant and microbial interactions with radionuclides is necessary, both to quantify the risks associated with heavy metal and radionuclide transfer through food chains, and to assess the potential for plant and microbial bioremediation practices.

Using the abandoned pitchblende mine of South Terras, Cornwall as a case study, the influence of arbuscular mycorrhizal fungi (AMF; a symbiotic association between a fungus and plant root) on plant lead $(\mathrm{Pb})$ and uranium (U) uptake and transfer has been studied. $\mathrm{Pb}$ was selected as it is abundant at the mine site, forms a divalent cation and is nonessential for plant nutrition, being analogous to $U$ in these aspects. Understanding the influence of AMF interactions on the fate of heavy metals and radionuclides is critical as existing knowledge is limited, despite AMF being able to colonise $80-90 \%$ of plant species $[1,2]$.

Mesocosms grown in controlled conditions are compared utilising a combination of microscopy, autoradiography, multi-elemental analysis, enzymatic assays and microdialysis techniques. This combination of data has helped to refine current understanding of $\mathrm{Pb}$ and $\mathrm{U}$ chemical uptake and transfer behaviour within the soil-fungi-plant system. Future work will use $\mu-\mathrm{XRF}$ and XANES/EXAFS techniques to uncover the speciation characteristics of $U$ within plant roots, and to better constrain the independent contribution of AMF to a plant's $\mathrm{U}$ and $\mathrm{Pb}$ uptake and translocation behaviour.

[1] Davies et al. (2018). Multiple environmental factors influence ${ }^{238} \mathrm{U},{ }^{232} \mathrm{Th}$ and ${ }^{226} \mathrm{Ra}$ bioaccumulation in arbuscular mycorrhizal-associated plants. Sci Total Environ. 640-641. 921-934.

[2] Ren, C. G. et al., (2019). Enhanced phytoremediation of uranium-contaminated soils by arbuscular mycorrhiza and rhizobium. Chemosphere. 217, 773-779. 